\title{
Methicillin resistant Staphylococcus aureus causing osteomyelitis in a tertiary hospital, Mwanza, Tanzania
}

Vitus Silago ${ }^{1}$, Martha F. Mushi ${ }^{1 *}$, Boniface A. Remi ${ }^{1}$, Alute Mwayi ${ }^{1}$, Stephen Swetala ${ }^{2}$, Conjester I. Mtemisika ${ }^{3}$ and Stephen E. Mshana'

\begin{abstract}
Background: Culture results of fluid/pus from sinuses or open wound are not reliable in establishing the causative agent of osteomyelitis due to the high chances of contamination of superficial contaminants. Bone fragments obtained during surgery have been recommended as ideal sample to establish pathogens causing osteomyelitis. This study investigated pathogens causing osteomyelitis among patients undergoing orthopedic surgical treatment at Bugando Medical Centre.
\end{abstract}

Methods: A cross-sectional hospital-based study was conducted from December 2017 to July 2018 among 74 patients with osteomyelitis who underwent surgical treatments at Bugando Medical Centre, Mwanza, Tanzania. Bone fragments were collected using sterile $10 \mathrm{ml}$ of in-house prepared brain heart infusion broth (Oxoid, UK) during surgery. Specimens were processed according to standard operating procedures within an hour of collection. Data were analyzed using STATA 13.0.

Results: The median age of study participants was 12 with inter quartile range of 8-20 years. The majority 45 (60.8\%) of participants were male. All 74 non-repetitive bone fragment specimens had positive culture, of which 17 had dual growth of bacteria resulting to 91 bacterial isolates. Out of 91 isolates, 63 (85.1\%) were Staphylococcus aureus (S. aureus) of which 18 (28.6\%) were confirmed to be methicillin resistant Staphylococcus aureus strains. Fever was significantly associated with Staphylococcal osteomyelitis (100\% vs. $79.6 \%, p=0.029)$.

Conclusion: About one third of cases of Staphylococcal osteomyelitis in the current study were caused by methicillin resistant Staphylococcus aureus. There is a need of tailoring antibiotic management of osteomyelitis based on culture and sensitivity results for the better treatment outcome of the patients.

Keywords: Staphylococcal osteomyelitis, Methicillin resistant S. aureus, Bugando Medical Centre, Mwanza, Tanzania

\footnotetext{
* Correspondence: mushimartha@gmail.com

${ }^{1}$ Microbiology and Immunology department, Weill Bugando School of

Medicine, Catholic University of Health and Allied Sciences, P. O. Box 1464,

Mwanza, Tanzania

Full list of author information is available at the end of the article
}

(C) The Author(s). 2020 Open Access This article is licensed under a Creative Commons Attribution 4.0 International License, which permits use, sharing, adaptation, distribution and reproduction in any medium or format, as long as you give appropriate credit to the original author(s) and the source, provide a link to the Creative Commons licence, and indicate if changes were made. The images or other third party material in this article are included in the article's Creative Commons licence, unless indicated otherwise in a credit line to the material. If material is not included in the article's Creative Commons licence and your intended use is not permitted by statutory regulation or exceeds the permitted use, you will need to obtain permission directly from the copyright holder. To view a copy of this licence, visit http://creativecommons.org/licenses/by/4.0/ The Creative Commons Public Domain Dedication waiver (http://creativecommons.org/publicdomain/zero/1.0/) applies to the data made available in this article, unless otherwise stated in a credit line to the data. 


\section{Introduction}

Osteomyelitis is an inflammation of the bone that can be localized to only one part of the bone such as bone marrow, periosteum, or cortex [1]. In few occasions, osteomyelitis can disseminate to the surrounding tissues [1]. Osteomyelitis can be classified by the location within the bone, extent of dissemination and source of infection [1]. Bacteria are the commonest cause of osteomyelitis with $S$. aureus implicated in more than $75 \%$ of cases. S. aureus spreads to the bones through blood (hematogenous osteomyelitis) or directly as a result of open fractures $[2,3]$.

Staphylococcal osteomyelitis is a major global health concern because of the increasing antimicrobial resistance. Treatment of osteomyelitis is always complex because it requires a well-coordinated team of radiologist, orthopedic surgeon, and infectious diseases specialist [4]. Culture of fluid/pus from sinuses or discharging ulcers is not reliable, thus leading to the dependence of imaging and high suspicion of a surgeon in managing osteomyelitic patients. To determine the causative pathogen implicating in osteomyelitis, a sample of choice is bone fragments because culture of fluid/pus from sinuses or open is always associated with superficial bacteria contaminants $[4,5]$. This study investigated the pathogens causing osteomyelitis among patients undergoing surgical treatment at Bugando Medical Centre, Mwanza-Tanzania for the purpose of generating data that can be used to devise antibiotic treatment guidelines.

\section{Methodology}

\section{Study design, participants, duration, setting and} specimen collection

It was a cross sectional hospital based study, conducted from December 2017 to July 2018 among 74 patients with osteomyelitis admitted for surgery at Bugando Medical Centre (BMC), Mwanza, Tanzania. BMC is a tertiary referral hospital with 1000 bed capacity, serving seven regions namely: Mwanza, Musoma, Simiyu, Shinyanga, Kagera, Kigoma, and Geita. The study included all patients with clinical diagnosis of osteomyelitis planned for orthopedic surgery. As part of routine clinical care at enrollment, body temperature was measured using a digital thermometer MDD 93/42/EEC, “0197" (Holding Corp. $\mathrm{GmbH}$ Hamburg) and all patients with body temperature above $37.5^{\circ} \mathrm{C}$ were termed as febrile [6]. During surgical procedures, bone fragments were collected and placed into sterile universal bottle containing $10 \mathrm{ml}$ of in-house brain heart infusion (BHI) broth (Oxoid, UK) [7] to increase the yield of pathogenic bacteria. Specimens were sent to Microbiology laboratory of the Catholic University of Health and Allied Sciences within $1 \mathrm{~h}$ of collection.

\section{Laboratory procedures}

In the laboratory, universal bottles with samples were gently shaken 10 times, and then incubated at $37^{\circ} \mathrm{C}$ for $6 \mathrm{~h}$ [7].
After $6 \mathrm{~h}$ of incubation, specimens were mixed gently and a loop full $(10 \mu \mathrm{l})$ was inoculated on sheep blood agar and MacConkey Agar followed by aerobic incubation at $37^{\circ} \mathrm{C}$ for $24 \mathrm{~h}$. Conventional biochemical identification tests: Gram stain, catalase and coagulase, DNase were used to identify Gram-positive bacteria while Triple sugar iron agar (TSI), Sulfur-Indole-Motility (SIM), Simmons citrate, urease, and oxidase were used to identify Gram-negative bacteria [8]. Antibiotics susceptibility testing was performed using Kirby-Bauer disc diffusion technique. Tetracycline 30 $\mu \mathrm{g}$, gentamicin $10 \mu \mathrm{g}$, ciprofloxacin $5 \mu \mathrm{g}$, clindamycin $2 \mu \mathrm{g}$, and vancomycin $30 \mu \mathrm{g}$ were used for Gram-positive bacteria while ampicillin $10 \mu \mathrm{g}$, trimethoprim-sulfamethoxazole $1.25 / 23.75 \mu \mathrm{g}$, tetracycline $30 \mu \mathrm{g}$, gentamicin $10 \mu \mathrm{g}$, ciprofloxacin $5 \mu \mathrm{g}$, amoxycillin-clavulanic acid $20 / 10 \mu \mathrm{g}$, ceftriaxone $30 \mu \mathrm{g}$, ceftazidime $30 \mu \mathrm{g}$, piperacillin-tazobactam 100/ $10 \mu \mathrm{g}$, and meropenem $10 \mu \mathrm{g}$ were used for Gram-negative bacteria [9]. Cefoxitin 30- $\mu$ g discs were used for detection of MRSA as per Clinical Laboratory Standard Institute (CLSI) recommendations [10].

Briefly, pure fresh colonies were suspended in sterile $0.85 \%$ normal saline to make a suspension equivalent to $0.5 \mathrm{McF}$ arland. Using sterile cotton swab, suspension was inoculated on the entire surface of Muller Hinton agar (MHA) plate; thereafter, antibiotic discs were seeded within $15 \mathrm{~min}$ of inoculation. MHA plates were incubated aerobically at $37^{\circ} \mathrm{C}$ for $18-24 \mathrm{~h}$. Clinical and Laboratory Standards Institute (CLSI-2016) was used to interpret zones of inhibitions [10].

\section{Quality control}

Staphylococcus aureus ATCC 25923 and Escherichia coli ATCC 25922 were used as control strains.

\section{Ethical considerations}

Protocol of this study was ethically approved by the joint CUHAS/BMC research ethics and review committee and given certificate number 470/2017. All participants signed informed consent and those aged below 18 years their parents/guardians' consented on their behalf. Laboratory results were timely reported to the attending physicians to guide rational antibiotics therapy.

\section{Results \\ Socio-demographic and clinical characteristics of participants}

A total of 74 osteomyelitic patients with median age of 12 and inter quartile range of 8-20 years were enrolled. Males made the majority $45(60.8 \%)$ of the study participants. The majority 66 (89.2\%) of participants had infections of the long bones. A total of 20 (27\%) and 32 (43.2\%) had fever and history of antibiotic use before the index admission, respectively. The median duration of antibiotic use was 2 weeks with inter quartile range of 
2-4 weeks. The most commonly used antibiotic was ampicillin-cloxacillin which was reported in 65.6\% (21/ 35) of patients (Table 1).

\section{Laboratory results}

All 74 non-repetitive samples yielded growth of pathogenic bacteria on culture. Seventeen samples had dual growth making a total of 91 isolates. The majority of isolates 74 (81.3\%) were Gram-positive bacteria. Of 17 patients with dual bacterial growth, 11(64.7\%) had Streptococcus pyogenes and S. aureus, 3(17.6\%) had Proteus vulgaris and Klebsiella pneumoniae, and 3(17.6\%) had Pseudomonas aeruginosa with either Klebsiella pneumoniae, S. aureus, or Citrobacter freundii. In general, the most frequently isolated bacteria was S. aureus $69.2 \%$ (63/91) followed by Streptococcus pyogenes 12.1\% (11/91) and Klebsiella pneumoniae 6.6\% (6/91) (Fig 1). Methicillin resistant Staphylococcus aureus (MRSA) strains were confirmed in 18 (28.6\%) of 63 Staphylococcus aureus isolates (Table 2).

\section{Susceptibility patterns}

Generally, Gram-positive bacteria were 100\%, 100\%, 98.7\%, $100 \%$, and $100 \%$ sensitive to gentamicin, ciprofloxacin, clindamycin, tetracycline, and vancomycin, respectively. Out of 63 S. aureus, 18 (28.6\%) were MRSA with 10 of MRSA strains being isolated from patients with history of antibiotic use.

The proportion of resistance among Gram-negative bacteria was as follows: ampicillin (100\%), amoxicillinclavulanic acid (100\%), ceftriaxone (66.7\%), ceftazidime (64.7\%), trimethoprim-sulfamethoxazole (41.7\%), tetracycline (41.7\%), piperacillin-tazobactam (35.5\%), gentamicin $(23.5 \%)$, ciprofloxacin $(0 \%)$, and meropenem $(0 \%)$ (Table 2).

\section{Factors associated with Staphylococcal osteomyelitis}

By chi-square test, only having fever was statistically associated with Staphylococcal osteomyelitis (100\% vs. $79.6 \%, p=0.029$ ) (Table 3). The sub-analysis by age (children vs. adults) found no significance difference terms of pathogens distributions and factors associated with Staphylococcus osteomyelitis (data not shown).

\section{Discussion}

Dependence on imaging in the management of osteomyelitis in resource limited settings [11] is associated

Table 1 Socio-demographic and clinical characteristics of study participants

\begin{tabular}{|c|c|c|c|}
\hline \multicolumn{2}{|l|}{ Characteristics } & \multirow{2}{*}{$\begin{array}{l}\text { Frequency }(N) \\
12\end{array}$} & \multirow{2}{*}{$\begin{array}{l}\text { Percentages }(\%) \\
8-20]\end{array}$} \\
\hline Median age (IQR) in years & & & \\
\hline \multirow[t]{2}{*}{ Sex } & Males & 45 & 60.8 \\
\hline & Females & 29 & 39.2 \\
\hline \multirow[t]{4}{*}{ Education level } & None formal education & 21 & 28.4 \\
\hline & Primary school & 32 & 43.2 \\
\hline & Secondary school & 15 & 40.3 \\
\hline & College and above & 6 & 8.1 \\
\hline \multirow[t]{2}{*}{ Referrals outside Mwanza } & No & 35 & 47.3 \\
\hline & Yes & 39 & 52.7 \\
\hline \multirow[t]{2}{*}{ Major complaint } & Sepsis & 32 & 43.2 \\
\hline & Trauma & 42 & 56.8 \\
\hline \multirow[t]{2}{*}{ Infected bone } & Long bone & 66 & 89.2 \\
\hline & Short bone & 8 & 10.8 \\
\hline \multirow[t]{2}{*}{ Infected site condition } & Discharging & 65 & 87.8 \\
\hline & Swelling & 9 & 12.2 \\
\hline \multirow[t]{2}{*}{ Fever during enrollment } & Yes & 20 & 27 \\
\hline & No & 54 & 73 \\
\hline \multirow[t]{2}{*}{ Pre-exposed to antibiotics } & Yes & 32 & 43.2 \\
\hline & No & 42 & 56.8 \\
\hline \multicolumn{2}{|c|}{ Median duration (IQR) of drug use in weeks } & 2 & {$[2-4]$} \\
\hline \multirow[t]{4}{*}{ Type of antibiotic exposed } & Ampicillin-cloxacillin (Ampiclox) & 21 & 65.6 \\
\hline & Ceftriaxone & 5 & 15.6 \\
\hline & Ampiclox with ceftriaxone & 4 & 12.5 \\
\hline & Herbal medicine & 2 & 6.3 \\
\hline
\end{tabular}


80

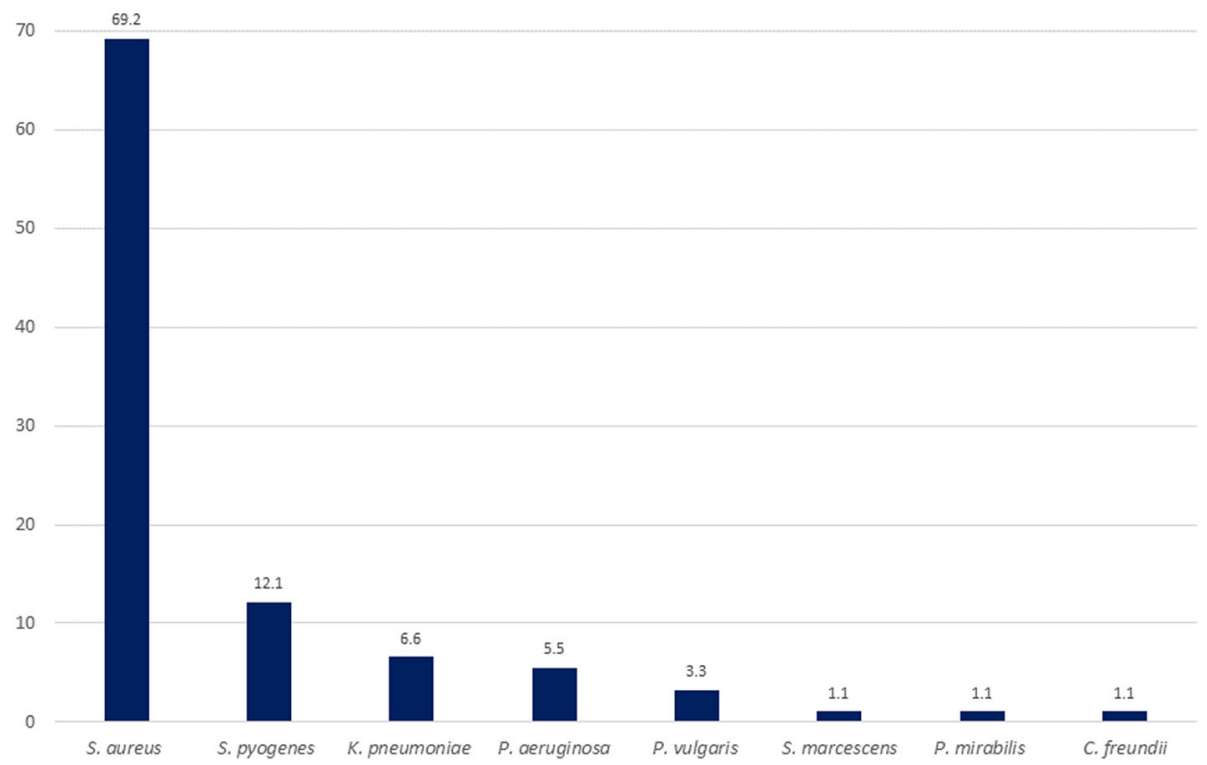

Fig. 1 Bacteria isolated in 74 patients with osteomyelitis

with poor prognosis of patients and morbidity such as amputation of infected bone. In the places where there is increased isolation of multidrug resistant bacteria, rational antibiotic therapy is mandatory for proper patients' management [12]. However, in many resource limited settings, there is no routine culture for the diagnosis of osteomyelitis. Good prognosis of patients with osteomyelitis depends much on proper identification of etiological agent and timely treatmen $\mathrm{t}[13]$.
There is limited data regarding the pathogens causing osteomyelitis from developing countries. The majority of participants in this study were adolescent males, with infection of long bones as a result of trauma. The majority of these infections had purulent drainage from infection sites as previously reported elsewhere [14-16]. The escalating burden of long bones fracture in Tanzania among adolescent males is highly associated with the legalization of motorcycles as a means public transport $[17,18]$. As

Table 2 Antibiotic susceptibility patterns of isolated bacteria

\begin{tabular}{|c|c|c|c|c|c|}
\hline \multirow[t]{2}{*}{ Antibiotic agents } & \multirow[t]{2}{*}{ Potency } & \multicolumn{2}{|c|}{ Gram positive bacteria } & \multicolumn{2}{|c|}{ Gram negative bacteria } \\
\hline & & Sensitive & Resistant & Sensitive & Resistant \\
\hline Ampicillin & $10 \mu \mathrm{g}$ & NA & NA & $0 \%$ & $100 \%$ \\
\hline Trimethoprim-sulphamethaxazole & $1.25 / 23.75 \mu \mathrm{g}$ & NT & NT & $58.3 \%$ & $41.7 \%$ \\
\hline Tetracycline & $30 \mu \mathrm{g}$ & $100 \%$ & $0 \%$ & $58.3 \%$ & $41.7 \%$ \\
\hline Gentamicin & $10 \mu \mathrm{g}$ & $100 \%$ & $0 \%$ & $76.5 \%$ & $23.5 \%$ \\
\hline Ciprofloxacin & $30 \mu \mathrm{g}$ & $100 \%$ & $0 \%$ & $100 \%$ & $0 \%$ \\
\hline Clindamycin & $10 \mu \mathrm{g}$ & $98.6 \%$ & $1.4 \%$ & NA & NA \\
\hline Cefoxitin (S. aureus only) & $30 \mu \mathrm{g}$ & $71.4 \%$ & $28.6 \%$ & NA & NA \\
\hline Vancomycin & $30 \mu \mathrm{g}$ & $100 \%$ & $0 \%$ & NA & NA \\
\hline Ceftriaxone & $30 \mu \mathrm{g}$ & NA & NA & $33.3 \%$ & $66.7 \%$ \\
\hline Ceftazidime & $30 \mu \mathrm{g}$ & NA & NA & $35.3 \%$ & $64.7 \%$ \\
\hline Piperacillin-tazobactam & $100 / 10 \mu g$ & NA & NA & $64.7 \%$ & $35.3 \%$ \\
\hline Amoxicillin-clavulanic acid & $20 / 10 \mu g$ & NA & NA & $0 \%$ & $100 \%$ \\
\hline Meropenem & $10 \mu \mathrm{g}$ & NA & NA & $100 \%$ & $0 \%$ \\
\hline
\end{tabular}

NT not tested, NA not applicable 
Table 3 Factors associated with Staphylococci osteomyelitis

\begin{tabular}{|c|c|c|c|c|c|}
\hline \multirow[t]{2}{*}{ Characteristics } & & \multicolumn{2}{|c|}{ Staphylococcal osteomyelitis } & \multirow[t]{2}{*}{$\mathrm{Chi}^{2}$} & \multirow{2}{*}{$\begin{array}{l}p \\
\text { value }\end{array}$} \\
\hline & & Positive $n(\%)$ & Negative $n(\%)$ & & \\
\hline \multirow[t]{2}{*}{ Age category } & Children & $44(89.8)$ & $5(10.2)$ & 0.963 & 0.124 \\
\hline & Adult & $19(76.0)$ & $6(24.0)$ & & \\
\hline \multirow[t]{2}{*}{ Sex } & Females & $26(89.7)$ & $3(10.3)$ & 0.7699 & 0.380 \\
\hline & Males & $37(82.2)$ & $8(17.8)$ & & \\
\hline \multirow[t]{2}{*}{ Referral outside Mwanza } & No & $30(85.7)$ & $5(14.3)$ & 0.0176 & 0.894 \\
\hline & Yes & $33(84.6)$ & $6(15.4)$ & & \\
\hline \multirow[t]{4}{*}{ Education level of participant } & Non-formal & $19(90.5)$ & $2(9.5)$ & 2.5445 & 0.467 \\
\hline & Primary level & $28(87.5)$ & $4(12.5)$ & & \\
\hline & Secondary level & $12(80.0)$ & $3(20.0)$ & & \\
\hline & College and above & $4(66.7)$ & $2(33.3)$ & & \\
\hline \multirow[t]{2}{*}{ Complaint } & Sepsis & $28(87.5)$ & $4(12.5)$ & 0.2492 & 0.618 \\
\hline & Trauma & $35(83.3)$ & $7(16.7)$ & & \\
\hline \multirow[t]{2}{*}{ Condition } & Discharging & $57(87.7)$ & $8(12.3)$ & 2.2615 & 0.097 \\
\hline & Swelling & $6(66.7)$ & $3(33.3)$ & & \\
\hline \multirow[t]{2}{*}{ Fever during enrollment } & Yes & $20(100.0)$ & $0(0.0)$ & 4.7854 & 0.029 \\
\hline & No & 43 (79.6) & $11(20.4)$ & & \\
\hline
\end{tabular}

previously reported [18-20], this study has confirmed that purulent discharge from the affected part of the bone is the commonest symptoms of osteomyelitis. The purulent discharge from the affected part of the bone increases the suspicious index of pyogenic infections and call for the need of microbiological investigations [21].

The $69.2 \%$ prevalence of the Staphylococcus osteomyelitis in the current study was certainly high. However, these results align with previous reports which reported $S$. aureus as a major pathogen causing osteomyelitis [22, 23]. It was observed that about one third of $S$. aureus isolates were MRSA which is almost double to what was observed 10 years ago in the same setting among $S$. aureus isolates from wounds [24, 25]. These findings cement the previous observations of increasing trend of MRSA in study settings made by Moremi et al. in 2016 [26].

Over the counter use of antibiotics and irrational empirical treatment of bacterial infections in the study settings [27] might result to the selection of resistant Staphylococcus aureus strains such as MRSA. This is further supported by the fact that in the current study the majority of patients with history of antibiotic use were using ampicillincloxacillin hence more likelihood of selecting MRSA strains.

The current study has observed that $S$. aureus isolates were highly susceptible to chloramphenicol, clindamycin, and vancomycin. This could be explained by the fact that these antimicrobial agents are not used in self-medication as penicillin, ampicillin, and amoxycillin. In addition, clindamycin and vancomycin are preserved as the second line treatment regimen [11]. The standard treatment guidelines in Tanzania recommend the use of ampicillin, tetracycline, and/or erythromycin in management of gram positive bacterial infection [11]. Based on these results, clindamycin should be considered as the first line empirical therapy while waiting for culture and sensitivity results, and when gram negative pathogens are suspected Piperacillin tazobactam can be added. These recommendations are supported by a previous systemic review [28].

Only fever was significantly associated with Staphylococcal osteomyelitis $(p=0.029)$. Fever is the commonest response of infection or inflammation whereby the interaction between exogenous pyogenic bacteria and the organum vasculosum of the lamina terminalis (OVLT) induces production of cytokines and increases synthesis of prostaglandin E2 (PGE2) resulting to body temperature rise [29].

\section{Limitation}

Due to limited diagnostic facilities, the study did not investigate all range of pathogens that may cause osteomyelitis such fungi, Mycobacteria spp., and anaerobic pathogens. In addition, data regarding involvement of the joint were not collected.

\section{Conclusion and recommendation}

This study found high prevalence of Staphylococcal osteomyelitis among patients underwent surgical treatment at Bugando Medical Centre with a third of these patients infected with MRSA. Fever was statistically significant associated with positive Staphylococcal osteomyelitis. There is a need to tailor antibiotic management of osteomyelitis based on culture and sensitivity patterns for the better outcome of the patients. 


\section{Abbreviations}

BHI: Brain heart infusion; BMC: Bugando Medical Centre; CLSI: Clinical Laboratory Standard Institute; MHA: Muller Hinton agar; MRSA: Methicillin resistant Staphylococcus aureus; OVLT: Organum vasculosum of the lamina terminalis; SIM: Sulfur-Indole-Motility; TSI: Triple sugar iron agar

\section{Acknowledgements}

Authors acknowledge the department of Microbiology and Immunology, Weill Bugando School of Medicine, Catholic University of Health and Allied Sciences, Bugando.

\section{Authors' contributions}

VS, SS, MFM, and SEM designed this study. BAL and SS collected data. VS, BAL, AM, and CIM performed laboratory procedures. VS, CIM, MFM, and SEM analyzed data. MFM, VS, and CIM wrote the first draft of manuscript. SEM critically revised the manuscript. All authors approved the final draft to be published.

\section{Funding}

No fund was received for this work.

\section{Availability of data and materials}

The data is available upon request and the request should be made to the Director of Research and Innovation, Catholic University of Health and Allied Sciences (vc@bugando.ac.tz).

\section{Ethics approval and consent to participate}

Protocol of this study was ethically approved by the joint CUHAS/BMC research ethics and review committee and given certificate number 470/ 2017. Only consented participants were enrolled in this study, participants aged below 18 years their mothers'/guardians' consented on their behalf. Laboratory results were timely reported to attending physicians to guide rational antibiotics therapy.

\section{Consent for publication}

None applicable

\section{Competing interests}

The authors declare that they have no competing interests.

\section{Author details}

${ }^{1}$ Microbiology and Immunology department, Weill Bugando School of Medicine, Catholic University of Health and Allied Sciences, P. O. Box 1464, Mwanza, Tanzania. ${ }^{2}$ Department of Surgery, Bugando Medical Centre, P. O. Box 1370, Mwanza, Tanzania. ${ }^{3}$ Central Pathology Laboratory, Bugando Medical Centre, P. O. Box 1370, Mwanza, Tanzania.

\section{Received: 29 October 2019 Accepted: 27 February 2020}

Published online: 05 March 2020

\section{References}

1. Kavanagh N, Ryan EJ, Widaa A, Sexton G, Fennell J, O'Rourke S, Cahill KC Kearney CJ, O'Brien FJ, Kerrigan SW. Staphylococcal osteomyelitis: disease progression, treatment challenges, and future directions. Clin Microbiol Rev. 2018;31(2):e00084-17.

2. Del Pozo EG, Collazos J, Carton J, Camporro D, Asensi V. Factors predictive of relapse in adult bacterial osteomyelitis of long bones. BMC Infect Dis. 2018;18(1):635

3. Carek PJ, Dickerson LM, Sack JL. Diagnosis and management of osteomyelitis. Am Fam Physician. 2001;63(12):2413-20.

4. Fritz JM, McDonald JR. Osteomyelitis: approach to diagnosis and treatment. Phys Sportsmed. 2008;36(1):50-4.

5. Jacob E, Durham L, Falk M, Williams T, Wheat L. Antibody response to teichoic acid and peptidoglycan in Staphylococcus aureus osteomyelitis. J Clin Microbiol. 1987:25(1):122-7.

6. Ergönül Ö, Willke A, Azap A, Tekeli E. Revised definition of 'fever of unknown origin': limitations and opportunities. J Infect. 2005;50(1):1-5.

7. Senneville E, Melliez H, Beltrand E, Legout L, Valette M, Cazaubie M, Cordonnier M, Caillaux M, Yazdanpanah Y, Mouton Y. Culture of percutaneous bone biopsy specimens for diagnosis of diabetic foot osteomyelitis: concordance with ulcer swab cultures. Clin Infect Dis. 2006; 42(1):57-62.

8. Winn WC. Koneman's color atlas and textbook of diagnostic microbiology: Lippincott Williams \& Wilkins; 2006

9. Bauer A, Kirby W, Sherris JC, Turck M. Antibiotic susceptibility testing by a standardized single disk method. Am J Clin Pathol. 1966;45(4_ts):493-6.

10. CLSI C. Performance standards for antimicrobial susceptibility testing: Clinical Lab Standards Institute; 2016.

11. Ministry for Health CD, Gender, Elderly and Children. Standard Treatment Guidelines \& National Essential Medicines List-Tanzania Mainland. 2017.

12. Allerberger $F$, Amann $S$, Apfalter $P$, Brodt $H-R$, Eckmanns T, Fellhauer $M$, Geiss $\mathrm{H}$, Janata $\mathrm{O}$, Krause $\mathrm{R}$, Lemmen $\mathrm{S}$. Strategies to enhance rational use of antibiotics in hospital: a guideline by the German Society for Infectious Diseases. Infection. 2016;44(3):395-439.

13. Zuluaga AF, Galvis W, Jaimes F, Vesga O. Lack of microbiological concordance between bone and non-bone specimens in chronic osteomyelitis: an observational study. BMC Infect Dis. 2002;2(1):8.

14. Caetano A, Nunes A, Pinheiro C, Tavares J, Bastos R, Cardoso C. Treatment of long-term chronic post-traumatic osteomyelitis of the tibia: two case reports. In: Orthopaedic Proceedings: 2015: The British Editorial Society of Bone \& Joint Surgery; 2015. p. 17.

15. Juutilainen V. Posttraumatic osteomyelitis. Suomen Ortopedia ja Traumatologia. 2011;34(1):38-41.

16. Vardakas K, Kontopidis I, Gkegkes I, Rafailidis P, Falagas M. Incidence, characteristics, and outcomes of patients with bone and joint infections due to community-associated methicillin-resistant Staphylococcus aureus: a systematic review. Eur J Clin Microbiol Infect Dis. 2013;32(6):711-21.

17. Chalya PL, Mabula JB, Ngayomela IH, Kanumba ES, Chandika AB, Giiti G, Mawala B, Balamuka D. Motorcycle injuries as an emerging public health problem in Mwanza City, Tanzania: a call for urgent intervention. Tanzan J Health Res. 2010;12(4):214-21.

18. Mutasingwa D, Aarø L. Injury registration in a developing country. A study based on patients' records from four hospitals in Dar es Salaam, Tanzania; 2001

19. Shrestha A, Sah SK. Chronic osteomyelitis on an old compound fracture. J Coll Med Sci Nepal. 2012;8(2):58-63.

20. Pande K. Optimal management of chronic osteomyelitis: current perspectives. Orthoped Res Rev. 2015;7:71-81.

21. Birt MC, Anderson DW, Toby EB, Wang J. Osteomyelitis: recent advances in pathophysiology and therapeutic strategies. J Orthopaed. 2017;14(1):45-52.

22. Olson ME, Horswill AR. Staphylococcus aureus osteomyelitis: bad to the bone. Cell Host Microbe. 2013;13(6):629-31.

23. Babaei S. Osteomyelitis septic arthritis \& soft tissue infections; 2013.

24. Mshana S, Kamugisha E, Miramb M, Chalya P, Rambau P, Mahalu W, Lyamuya E. Prevalence of clindamycin inducible resistance among methicillin-resistant Staphylococcus aureus at Bugando Medical Centre, Mwanza, Tanzania. Tanzania J Health Res. 2009;11:2.

25. Moremi N, Mushi MF, Fidelis M, Chalya P, Mirambo M, Mshana SE. Predominance of multi-resistant gram-negative bacteria colonizing chronic lower limb ulcers (CLLUs) at Bugando Medical Center. BMC Res Notes. 2014; 7(1):211.

26. Moremi N, Claus H, Mshana SE. Antimicrobial resistance pattern: a report of microbiological cultures at a tertiary hospital in Tanzania. BMC Infect Dis. 2016;16(1):756.

27. Horumpende PG, Sonda TB, van Zwetselaar M, Antony ML, Tenu FF, Mwanziva CE, Shao ER, Mshana SE, Mmbaga BT, Chilongola JO. Prescription and non-prescription antibiotic dispensing practices in part I and part II pharmacies in Moshi Municipality, Kilimanjaro Region in Tanzania: A simulated clients approach. PloS One. 2018:13(11):e0207465.

28. Spellberg B, Lipsky BA. Systemic antibiotic therapy for chronic osteomyelitis in adults. Clin Infect Dis. 2011:54(3):393-407.

29. Walter EJ, Hanna-Jumma S, Carraretto M, Forni L. The pathophysiological basis and consequences of fever. Crit Care. 2016;20(1):200.

\section{Publisher's Note}

Springer Nature remains neutral with regard to jurisdictional claims in published maps and institutional affiliations. 